\title{
A contact tracing prospective cohort retrieving epidemiological facts on SARS-CoV-2 transmission aspects; a serological analysis
}

\section{Reza Vazirinejad}

Rafsanjan University of Medical Sciences

Parvin Khalili ( $\sim$ parvinkhalili61@yahoo.com)

Rafsanjan University of Medical Sciences

Abdollah Jafarzadeh

Kerman University of Medical Sciences

\section{Ziba Shabani}

Rafsanjan University of Medical Sciences

\section{Ahmad Jamalizadeh}

Rafsanjan University of Medical Sciences

\section{Batool Rezaei}

Rafsanjan University of Medical Sciences

Hassan Ahmadnia

Hamedan University of Medical Sciences

Mohammad-taghi Rezayati

Rafsanjan University of Medical Sciences

Mohammad Ebrahimian

Rafsanjan University of Medical Sciences

Gholamreza Mehralinasab

Rafsanjan University of Medical Sciences

\section{Azam Bagherizadeh}

Rafsanjan University of Medical Sciences

\section{Shima Bazaz}

Rafsanjan University of Medical Sciences

\section{Erfan Vazirinejad}

University of Pecs

\section{Research Article}

Keywords: SARS-CoV-2, coronavirus, infection, spread, serological 
Posted Date: December 23rd, 2020

DOI: https://doi.org/10.21203/rs.3.rs-121829/v1

License: (c) (i) This work is licensed under a Creative Commons Attribution 4.0 International License. Read Full License 


\section{Abstract}

Introduction Novel coronavirus spread seems mysterious enough for convincing us to double check the indices being used to predict its transmission. Serological analysis was applied for assessing some metric epidemiological aspects of the infection and its transmissibility among people who were in contact with SARA-CoV-2 patients.

Methods In this contact tracing prospective cohort study, 453 contact cases of forty COVID 19 patients were followed for three months. SARS-CoV-2 patients were diagnosed by real time polymerase chain reaction testing of nasopharyngeal samples. The history of infectiousness was detected by serological testing of IgG and IgM. Trained expert team completed two questionnaires and blood samples were taken by experts in laboratory. Data were analyzed using SPSS (Ver.21) and R software. Some important epidemiological characteristics of the infection were calculated.

Results Mean age of SARS-CoV-2 patients and contact cases were $53.0 \pm 18.2$ and $30.8 \pm 19.3$ years, respectively. Overall $\mathrm{R}_{0}$ of the infection was 2.56 . Household and non-household secondary attack rates (SAR) were $20 \%(95 \% \mathrm{Cl} ; 12.7-27.3)$ and $11.3 \%(95 \% \mathrm{Cl} ; 6.1-16.5)$, respectively. Transmission probability in each contact was 0.0205 and the serial interval was $6.4 \pm 4.6(95 \% \mathrm{Cl} ; 5.2-7.6)$ days. SAR among contact cases who exposed asymptomatic primary cases $(28 \%, 95 \% \mathrm{Cl} ; 10-46 \%)$ was higher than that (13.8\%, 95\% Cl;9.4-18.2) among contact cases exposing to symptomatic patients.

Conclusions We concluded a herd immunity between 60 and $65 \%$ is needed in human communities. Findings demonstrated how much reduction in infection $\mathrm{R}_{0}$ is predicted based on both clinical and public health interventions.

\section{Introduction}

About 10 months passed from the date when the first case of the new coronavirus (SARS-CoV-2) infection was reported from Wuhan, China. The first World Health Organization (WHO) report for COVID 19 was issued on January 21, 2020 [1]. As of the date, the disease is in the form of pandemic as almost all countries around the world are contaminated and no vaccine and specific treatment are available, yet.

At the beginning weeks of the infection appearance, there was concern about some important aspects of the infection transmission. For instance, Burki reported on February 17, 2020 from the president of the American Society of Tropical Medicine and Hygiene, that "My sense is that asymptomatic transmission is not the major motor of this outbreak". She also reported that "the extent to which asymptomatic and subclinical patients can pass on the virus still remains unclear" [2]. Although many epidemiological investigations have been conducted in the last few months, there is not enough findings to provide accurate evidence for policy makers to answer these questions.

Similar to any other infection, every detail about novel coronavirus transmissibility can play a crucial role in programs of controlling the spread of the infection. Household contacts between people are closer 
than outdoor contacts and the probability of transmission should clearly be different. The type and the duration of contacts are also important and should be taken into account. Accurate data of contact tracing should be collected to provide the most exact information about human-to-human transmissibility of the infectious agent. Household Secondary Attack Rate (SAR) of SARS-CoV-2 was measured in few studies such as Jing et al study [3].

Winter and Hegde commented that in case of infectious pathogens, in order to contact tracing in highly dense populations, serological analysis can be useful [4]. However, cross-reactivity of serological tests with other viral pathogens is criticized. Serological analysis along with contact tracing is also useful to estimate the proportion of asymptomatic infectors in the population [4]. Serological testing can have a crucial role in identifying convalescent cases or people with milder disease who might have been missed by other surveillance methods [5].

Bi et al reported some metric measures of COVID 19 transmission including $\mathrm{R}_{0}$ and SAR among a group of patients and their contacts [6]. The accuracy of findings about the SARS-CoV-2 infection characteristics reported by authors who were in rush to publish their results at the first months of the infection appearance in high impact journals might be criticized. In our contact tracing prospective cohort, serological analysis was applied to have more accurate data for assessing some metric epidemiological measures of the infection and its transmissibility among people who were in contact with SARA-CoV-2 patients.

\section{Material And Methods}

Setting, participants and data collection

In this prospective cohort study, all patients with confirmed COVID 19 infection whose disease was confirmed using RT-PCR test, during March 1, 2020 to April 30, 2020, (Rafsanjan County, Kerman Province, Southeast, Iran) were invited $(n=48)$. All contact cases (household and non-household) were traced for 3 months $(n=453)$ and some details about the date, type, place and duration of their exposure to 40 (8 were excluded due to their opposition) COVID 19 patients (as primary cases) were registered by trained experts (figure 1). The ethics committee of Rafsanjan University of Medical Sciences approved this study before data collection (Ethical code: IR.RUMS.REC.1399.001)

At the first phase of the study, demographic characteristic of patients $(n=48)$ along with information about their medical situation were recorded by protected trained expert when they were admitted in hospital, after receiving patients (or relatives) written consent. Some extra items were also asked from patients about their contacts during; 1- the time before the first symptom of COVID 19 (at least for two weeks based on the COVID 19 incubation period), 2- the time period between the first symptom onset and admission in hospital, and 3- the duration between patients' admission and the time of data collection.

In the second phase, the research team followed all people who were listed as contact cases of each COVID 19 patients during the three months (total=453). Contact cases were visited in their living places 
by protected trained experts. Details about methods and objectives of the study were presented and participants consent was obtained from those who were willing to help with the study, before data collection. In case of contact cases younger than 18 years, we received the written informed consent form their parents before collecting data and introducing them to the reference laboratory for having their blood sample. In addition, this study was performed in accordance with the guidelines for the report of observational studies in epidemiology (STROBE).

\section{Outcomes}

Data were recorded on a checklist by trained experts, asking about age, gender, living place, nationality, occupation, educational status, marital status and family size. Some details about the diseases that participants might being suffered from, as co-morbidity (including hypertension, cardiovascular, respiratory diseases, diabetes, cancer and...) were also collected. Specific items about the date of contact (to the COVID 19 patient), the duration (minutes), place (indoors at home, indoors outside home such as shop or in a bus, and outdoors), the type (close such as kissing and/or hugging, not close not far such as hand shaking or having food together, and far such as contact with a distance more than 2 meters and/or for less than 2 minutes), and the number of contacts were asked. In the last section of the checklist some items were asked about the symptoms of the disease among contact cases, starting date of the first symptom, the duration of symptom, and the date of admission to hospital (if there was).

Further, all contact cases were also given a letter to reffer to the medical school laboratory and a $5 c c$ vein blood sample was taken from the top of forearm. The blood sample was used to measure CBC, ESR, CRP, $\lg G$ and $\lg M$. Serological tests were performed for assessing the presence level of the specific $\lg G$ and IgM antibodies. There was no cost for participants and the laboratory results were given to them for free. One member of our research team was requested to consult those participants whose laboratory results showed need further medical attention (an infection diseases specialist, consultant).

\section{Case definition;}

In our study, A primary confirmed case was a symptomatic or asymptomatic case with positive detection of SARS-CoV-2 nucleic acid by Real-time Polymerase Chain Reaction, RT-PCR, using specimens taken from respiratory excretions, or viral genes that are highly homologous to SARS-CoV-2 by sequencing method. In contact tracing, an individual with serology test results of $\lg G$ and $\lg M \geq 1$, with or without clinical symptoms, was defined as a secondary case.

\section{Statistical analysis;}

Standard methods based on the type and scale of measured variables were applied using SPSS (version 21). Charts and tables as well as descriptive statistical indices such as mean and median were used to 
present data. Parametric (t- test, ANOVA, correlation and regression) and non-parametric (Chi square, Fisher Exact, Mann Whitney $\mathrm{U}$ test and Kruskal Wallis) statistical tests were used for comparing different groups based on the distribution and deviation characteristics of the data. R statistical software was used to estimate basic reproductive numbers $\left(R_{0}\right)$ based on the details obtained from contact tracing data, and to fit predictive models.

We considered maximum mean duration of 7 days based on previous publications which reported mean SARS-CoV-2 incubation period of 5.5 and a maximum of 14 days [7-8]. Mean duration of symptoms presentation among patients $(n=48)$ in our study was calculated (Mean =6.4, Sd=6.6, Min=1, Max=30). Our search did not find any measure reported for the convalescent duration of COVID 19 (as of August 15, 2020), and we assumed at most 7 days for this stage of infection, giving an overall duration of 21 days of novel coronavirus infectiousness. This estimation is similar to what is reported by Long et al. Long et al reported the median duration of viral shedding in the asymptomatic COVID 19 Patients as 19 days (interquartile range (IQR), 15-26 d) [9]. Further, Wu et al reported a median disease duration of 22 (IQR, 18-26) days for SARS-CoV-2 patients [10].

We divided our participants' contacts based on their social relationship (living status) as household and non-household. We calculated probabilities of transmission for each household contact (people who were living with primary case in one house) and each non-household contact (people who were not living with the primary case in the same house).

Our model calculated the basic reproductive number based on aforementioned contact characteristics and $95 \%$ confidence interval was also reported. The results also include household and non-household SARs of the infection (SARS-CoV-2).

Regarding study objectinves, some characteristics of SARS-CoV-2 infection were calculated including the proportion of COVID 19 asymptomatic secondary cases, the infection serial interval, the probability of transmission per each contact, and the local average number of contacts of each primary case.

\section{Results}

Table 1 shows some characteristics of participants in the two groups of SARS-CoV-2 patients (primary cases, $n=48)$ and their contact cases $(n=453)$ in groups of household and non-household. Forty-eight laboratory confirmed SARS-CoV-2 infections were detected in the only university hospital of Rafsanjan (local population $=320,000)$, (March and April 2020), among whom $9(18.8 \%)$ were asymptomatic. Mean age of patients and contact cases were 53.0 \pm 18.2 (median = 50.5, Min=25, Max=95), and 30.77 \pm 19.3 (median $=31$, Min=1, Max=91) years, respectively. All contact cases of 40 primary cases were followed for three months beginning from each SARS-CoV-2 patient laboratory diagnosis date. Contact tracing identified 453 contact cases including the two groups of 206 household and 247 non-household contact cases. Among primary cases $(n=48)$, the first most common symptoms were cough $(10,21 \%)$, fever and 
chills $(10,21 \%)$. First symptom of SARS-CoV-2 in one primary case was eye irritation (1, 2\%). Nine (18.8\%) SARS-CoV-2 primary cases were asymptomatic (Table 2).

\section{The number of primary cases contacts;}

Our results showed that some contact cases were permanently living with a primary case. We counted such contact case as only one permanent contact per day. Contacts were recorded based on the three stages of $; 1$ - incubation period $=7$ days, 2 - illness period $=6.4 \approx 7$ days and 3 - convalescent period $=7$ days. Overall number of contacts during incubation period was 1348. Since, data of 63 contact cases was not, completely recorded, we divided the overall number of contacts to 34.5 instead of 40 primary cases. The mean number of contacts was 39.1 giving $5.6(39.1 / 7=5.6 \approx 6)$ daily contacts for each primary case during the incubation period. The number of daily contacts in the illness period and convalescent period were estimated similarly, applying the same method, giving $5.6(\approx 6)$ and $5.4(\approx 6)$ contacts per day, respectively.

\section{SARS-CoV-2 household and non-household SARs;}

Further, household and non-household SARs among contact cases along with 95\% confidence interval are reported in table 3. Our results of serology analysis (IgG antibody) showed an overall attack rate of $15.3 \%$ (95\% Cl; 10.9 - 19.7). Our results demonstrated that the SAR increased when the age of contact cases increased (Table 4). Household and non-household SARs of SARS-CoV-2 infection were $20 \%$ $(95 \% \mathrm{Cl} ; 12.7-27.3)$ and $11.3 \%(95 \% \mathrm{Cl} ; 6.1$ - 16.5), respectively. The risk of infection transmission among household contact cases was $1.41(\mathrm{OR}=1.41,95 \% \mathrm{Cl} ; 0.96-2.1)$ times higher than that risk among non-household contact cases.

\section{The proportion of asymptomatic COVID 19 infections;}

Among traced contact cases with serology results $(n=257), 18$ secondary cases were infected without any symptoms giving about $9.5 \%$ of contact cases who were asymptomatic. On the other hand, this group of secondary cases $(n=18)$, consist $46 \%$ of all infected secondary cases.

Out of 257 participants with the results of serology analysis, 25 were exposed to asymptomatic primary cases of which $7(28 \%, 95 \% \mathrm{Cl} ; 10-46)$ were IgG positive. whereas, among 232 participants exposing to symptomatic primary cases, there were 32 (13.8\%, 95\%Cl;9.4- 18.2) IgG positive participants. Transmission risk of SARS-CoV-2 among contact cases exposing to asymptomatic patients was significantly higher than that among contact cases exposing to symptomatic primary cases $(\mathrm{OR}=2.3, \mathrm{Cl}$ : $1.01-4.11)$. 
How long antibodies against SARS-CoV-2 infection may last?

Mean duration past from exposure (contact to primary cases) between the two groups of contact cases with ( $\geq 1)$ and without $(<1) \operatorname{lgG}$ antibodies ( $40.5 \pm 15.9$ and $36.9 \pm 15.2$, respectively) were not significantly different. There was also no significant correlation between the titer of IgG and the time past from the exposure among contact cases showing that antibodies against SARS-CoV-2 infection would remain for a long time in the serum of recovered people.

SARS-COV-2 serial interval;

The time distance between the date of the first symptom of primary cases (SARS-CoV-2 patients) and the date of the first symptom of secondary cases was measured. Mean SARS-CoV-2 serial interval was $6.4 \pm 4.6(95 \% \mathrm{Cl} ; 5.21-7.6)$ days with a median of 5 days (Min=1, Max=17 days).

Comorbidity effect on SARS-CoV-2 transmission;

There was no higher risk of becoming infected by SARS-CoV-2 infection due to having comorbidity as the two proportions of people with IgG $\geq 1$ among participants with and without comorbidity $13.7 \%$ and $15.5 \%$, respectively) were not statistically different. However, among traced contact cases with serology results ( $n=257), 19$ were suffering from hypertension and were more probable to have positive results of IgG $(31.6 \%, n=6)$ comparing to participants who did not reported this health problem $(13.9 \%, 33$ out of 238) $(\mathrm{OR}=2.9,95 \% \mathrm{Cl} ; 1.02-8.7)$.

Novel coronavirus transmission probability per each contact;

In order to measure the probability of infection transmission per each contact $\left(\mathrm{P}_{t}\right)$, the number of contacts with primary cases among participants with serological analysis was registered. The number of contacts were recorded based on the three stages of SARS-CoV-2 patients infectiousness; 1 - incubation period $\left(\mathrm{N}_{\text {inc }}\right)=645$ (7 days), 2- illness period $\left(\mathrm{N}_{\text {ill }}\right)=644\left(6.4 \approx 7\right.$ days), and 3- convalescent period $\left(\mathrm{N}_{\text {con }}\right)$ $=613$ (7 days). Also, the number of infected secondary cases $\left(N_{\text {sec }}\right)=39$.

$$
P_{t}=N_{\text {sec }} / N_{\text {inc }}+N_{i l l}+N_{\text {con }}
$$

$P_{t}=39 / 645+644+613=0.0205$

The overall probability of SARS-CoV-2 transmission per each contact is about 0.0205 . The probability of SARS-CoV-2 transmission per each household and non-household contacts were 0.0161 and 0.0337 , respectively.

The basic reproductive number of SARS-CoV-2 infection $\left(R_{0}\right)$; 
Regarding aforementioned indices derived from our results, it was possible to fit suitable models to predict SARS-CoV-2 infection spread in human populations. We used statistical software of $\mathrm{R}$ to provide models.

$R_{0}=P * C * D$

Where, $\mathrm{P}, \mathrm{C}$ and $\mathrm{D}$ stand for the probability of transmission per each contact, the number of contacts per unit time (day) and the duration of SARS-CoV-2 infectiousness, respectively.

Figure 2 shows, regression models illustrating the extend of which the value of $\mathrm{R}_{0}$ depends on the number of household and non-household daily contacts among contact cases. This model predicts the size of change in $\mathrm{R}_{0}$ value when the number of daily contacts to infected cases decreases in household and nonhousehold contact cases.

Our data also demonstrated the size of reduction in $\mathrm{R}_{0}$ value according to decreasing the duration of symptoms (illness period) among infected cases (figure 3 ).

\section{Discussion}

The most common symptoms at onset of illness were similar to what is reported by many other publications [11-13]. Interestingly, the first symptom of the infection in one of our patients was eye irritation and nine (18.8\%) SARS-CoV-2 patients (out of 48 ) were asymptomatic.

Close follow up of primary cases and their contact cases in this survey helped us to calculate almost accurate values for both the number of contact cases and the number of contacts for each primary case. The accuracy of these values was important in prediction of infection spread in the community as well as the extent of the herd immunity we need to control the spread of the SARS-CoV-2 infection.

As it is shown, serological analysis could help with contact tracing in urban regions and also useful for estimating the proportion of asymptomatic infected individuals [4]. Secondary cases in our study were detected based on the serum level of IgG specific antibody of 1 or higher. Suhandynata demonstrated high positive prediction value of IgG and IgM serologic results detecting real SARS-CoV-2 secondary cases [14].

Attack rate of SARS-CoV-2 infection in our study was 15.3\% (household and non-household secondary attack rates were $20 \%$ and $11.3 \%$, respectively). This finding is close to the results reported by Jing et al. ${ }^{3}$ Wanga et al reported a secondary transmission rate among household contacts of SARS-CoV-2 patients of $30 \%$ [15]. A systematic review conducted by Shah et al, presented a list of COVID 19 secondary attack rates reported in different regions of related surveys with a very wide range (minimum of $6 \%$ in South Africa a maximum of $49.56 \%$ in East Asia and Pacific) [16]. Our results as the first report of its type in Middle East, is based on serological analysis, whereas most of attack rates reported by Shah et al are estimated based on different methods. Also, Moscola et al reported 13.7\% (95\% Cl, 13.4\%-14.0\%) of US 
health care personnel were seropositive. However, this result might be unreliable to be compared with our results for which we assure that contact cases in our study were definitely in contact with infected primary cases. Their results also showed that only about $94 \%$ of PCR positive personnel were seropositive illustrating high sensitivity of our serology analysis [17].

What we found about the proportion of contact cases who might convert to asymptomatic secondary cases plays vital role to fit models for predicting the future spread of SARS-CoV-2 infection in the community. Our findings showed that about $10 \%$ (9.5\%) of all contact cases become infected and generates antibodies against SARS-CoV-2 infection without any symptoms. Asymptomatic cases also consist $46 \%$ of all secondary cases based on our results. This shows $46 \%$ of secondary cases who were infected showed no symptoms and could spread the novel coronavirus into the community without laboratory detection. This is suitable explanation to the new wave of the epidemic in many countries. If we put the attack rate equal to 15.3, beside this finding, there might be proper explanation for the situation of spreading the novel coronavirus in human communities, so far. Asymptomatic cases spread SARS-CoV-2 infection in communities, without detection. Shah et al concluded that asymptomatic cases in comparing to symptomatic cases, have a lower risk of spreading the infection [16], whereas this is not in concordance with our results. We showed that secondary attack rate among contact cases exposed to asymptomatic primary cases $(28 \%)$ was significantly higher $(\mathrm{OR}=2.3, \mathrm{Cl}: 1.01-4.11)$ than that among contact cases exposing symptomatic cases (13.8\%). However, what is reported by Long et al, justifies our results [9]. Therefore, health policy makers would pay even more attention to asymptomatic novel coronavirus infections in terms of controlling the pandemic. Further, the titer of serum antibodies against SARS-CoV-2 in asymptomatic and non-asymptomatic cases was not significantly different, in our survey.

As of today (August 20,2020), no data is reported to clarify the duration of immunity after infection recovery. Although, in our study contact cases were followed for at most three months, our result could showed natural acquired active immunity against SARS-CoV-2 infection would protect recovered cases for a considerable duration.

In our study, SARS-CoV-2 serial interval was 6.4 (Median=5) days. Nishiura et al, reported the mean and the median serial interval for SARS-CoV-2, as $4.7 \pm 2.9$ and 4 days, respectively [18]. Similar to the results of Nishiura et al, median serial interval in our survey is shorter than mean incubation period $(7.32 \pm 6.7$ days) illustrating that transmission was, often, taken place before the onset of symptoms (i.e. during incubation period).

Based on our results, those contact cases who were suffering from hypertension, were at, significantly higher risk of SARS-CoV-2 infection ( $\mathrm{OR}=2.9,95 \% \mathrm{Cl} ; 1.02-8.7)$. Although, many studies confirmed higher risk of mortality due to SARS-CoV-2 infection among patients who also were suffering from hypertension, diabetes, cardiovascular diseases, and etc, as a comorbidity [19-20], no survey reported increased risk of becoming infected by novel coronavirus due to comorbidity. However, our results suggest more investigation should be conducted to shed a light on it. 
Accuracy of the probability of SARS-CoV-2 infection transmission estimated in our survey play vital role in fitting the model of predicting the novel coronavirus spread in the community. We estimated the amount of $R_{0}$ of SARS-CoV-2 infection which was 2.58. Based on this value of $R_{0}$, between 60 to $65 \%$ of the population should be immune (as the herd immunity) for controlling the epidemic. Although human communities' characteristics have profound effect on $R_{0}$, and a wide range of $R_{0}$ is reported by different authors, our estimation is very close to the results of Wu et al who reported an estimation of 2.68 (95\% $\mathrm{Crl}$ 2.47-2.86) for SARS-CoV-2 infection $R_{0}$ in China [21].

Direct association between $\mathrm{R}_{0}$ value and the two factors of the number of daily contacts and the duration of illness period helps health policy makers to estimate the effectiveness of their actions for controlling the spread of SARS-CoV-2 infection.

Although our findings shed more light on the specific characteristics of SARS-CoV-2 infection, some limitations exist. First, we have no idea about the probability of secondary cases detection which might be the serological result of false positive, or missing cases with false negative serology results, which could lead to overestimation or underestimation of the indices. Second, as long as there was no clear report of the duration of convalescent period, we considered a mean of 7 days, based on our experience and the consultation we received from others, for this stage of the infection.

\section{Conclusions}

We concluded a herd immunity between 60 and $65 \%$ is needed in human communities. Findings demonstrated how much reduction in infection $\mathrm{R}_{0}$ is predicted based on both clinical and public health interventions.

\section{Declarations}

\section{Acknowledgments}

The Iranian Minister of health and Medical Education, Rafsanjan University of Medical Sciences, and Social Determinants of Health Research Center of the university supported this survey, financially. We thank all participants in the two groups of SARA-CoV-2 patients and their contact cases who accepted to help with the survey.

\section{Authors' contributions}

PK, AJ, GM, and BR collected the data. RV, HA, and PK conceived the statistical analysis plan. HA and PK did statistical analyses under the supervision of RV. RV and EV drafted the manuscript. RV, AJ and ZS contributed to interpretation of results and writing of the manuscript. MR, SB, AB and ME conducted serological analysis and controlled related data.

\section{Funding}


This study has been supported by the Vice Chancellery for Research \& Technology of Rafsanjan University of Medical Sciences. The context of this article are the views of the authors and the funder had no role in design of the study and collection, analysis, and interpretation of data, decision to publish and writing the manuscript.

\section{Availability of data and materials}

Data sharing requests should be directed to professor R Vazirinejad (rvazirinejad@yahoo.co.uk)

\section{Ethics approval and consent to participate}

The ethics committee of Rafsanjan University of Medical Sciences approved this study before data collection (Ethical code: IR.RUMS.REC.1399.001). Written informed consent was obtained from the participants. In case of contact cases younger than 18 years, we received the written informed consent form their parents before collecting data and introducing them to the reference laboratory for having their blood sample. The data of Participants kept confidential and was only accessible to the study investigators.

\section{Consent for publication}

Not applicable

\section{Competing interests}

The authors declare that they have no competing interests.

\section{References}

1. World Health Organization. Novel Coronavirus (2019-nCoV), Situation Report-1, 21 JANUARY 2020.

2. Burki T. Outbreak of coronavirus disease 2019. Lancet Infect Dis. 2020 Mar; 20(3): 292-293.

3. Jing QL, Liu MJ, Zhang ZB, Fang LQ, Yuan J, Zhang AR, et al. Household secondary attack rate of COVID-19 and associated determinants in Guangzhou, China: a retrospective cohort study. Lancet Infect Dis 2020 Published Online June 17, 2020 https://doi.org/10.1016/ S1473-3099(20)30471-0

4. Winter AK, Hegde The important role of serology for COVID-19 control. The Lancet Infectious Disease. July 2020, 20 (7), 755-874. https://doi.org/10.1016/ S1473-3099(20)30322-4 (accessed July 11, 2020).

5. Yong SEF, Anderson DE, Wei WE, Pang J, Chia WN, Tan CW, et al. Connecting clusters of COVID-19: an epidemiological and serological investigation. Lancet Infect Dis 2020; 20: 809-15 https://doi.org/10.1016/ S1473-3099(20)30273-5.

6. Bi Q, Wu Y, Mei S, Ye C, Zou X, Zhang Z, et al. Epidemiology and transmission of COVID-19 in 391 cases and 1286 of their close contacts in Shenzhen, China: a retrospective cohort study. Lancet Infect Dis 2020. Published Online April 27, 2020 https://doi.org/10.1016/ S1473-3099(20)30287-5 
7. Lauer SA, Grantz KH, Bi Q, Jones FK, Zheng Q, Meredith HR, et al. The Incubation Period of Coronavirus Disease 2019 (COVID-19) From Publicly Reported Confirmed Cases: Estimation and Application. Ann Intern Med. 2020; doi:10.7326/M20-0504

8. Bouadma L, Lescure FX, Lucet JC, Yazdanpanah Y, and Timsit JF. Severe SARS-CoV-2 infections: practical considerations and management strategy for intensivists. Intensive Care Med. Published 26 Feb 2020, https://doi.org/10.1007/s00134-020-05967-x

9. Long QX, Tang XJ, Shi QL, Li Q, Deng HJ, Yuan J, et al. Clinical and immunological assessment of asymptomatic SARS-CoV-2 infections. Nature Medicine, published 18 June 2020, available at https://doi.org/10.1038/ s41591-020-0965-6.

10. Wu F, Liu M, Wang A, Lu L, Wang Q, Gu C, et al. Evaluating the Association of Clinical Characteristics With Neutralizing Antibody Levels in Patients Who Have Recovered From Mild COVID-19 in Shanghai, China. JAMA Internal Medicine. JAMA Intern Med.Published online August 18, 2020. doi:10.1001/jamainternmed.2020.4616

11. Huang C, Wang Y, Li X, Ren L, Zhao J, Hu Y, et al. Clinical features of patients infected with 2019 novel coronavirus in Wuhan, China. Lancet 2020; 395: 497-506. https://doi.org/10.1016/ S01406736(20)30183-5.

12. Shu-Cheong D, Wong PC, Wang C. SARS: clinical features and diagnosis. Respirology (2003) 8, S20S24.

13. Park SE. Epidemiology, virology, and clinical features of severe acute respiratory syndrome coronavirus-2 (SARS-CoV-2; Coronavirus Disease-19). CEP, 2020; 63(4), 119-124. https://doi.org/10.3345/cep.2020.00493

14. Suhandynata RT, Hoffman MA, Kelner MJ, McLawhon RW, Reed SL, and Fitzgerald Longitudinal Monitoring of SARS-CoV-2 IgM and IgGSeropositivity to Detect COVID-19. J Appl Lab Med. 2020 May 19 : jfaa079. Published online 2020 May 19. doi: 10.1093/jalm/jfaa079

15. Wanga Z, Ma WB , Zhengc $X$, Wu G, and Zhang Household transmission of SARS-CoV-2. Journal of Infection. 2020, 81, pp 179- 182.

16. Shah K. Saxona D. Mavalankar D. Secondary Attack Rate of COVID-19 in household contacts: Systematic review. QJM: An International Journal of Medicine, July 2020, DOI:1093/qjmed/hcaa232

17. Moscola J, Sembajwe G, Jarrett M, Farber B, Chang T, McGinn T, etal. Prevalence of SARS-CoV-2 Antibodies in Health Care Personnel in the New York City Area. JAMA September 1, 2020 Volume 324, Number 9; 893-895.

18. Nishiura H, Lintona NM, Akhmetzhanov AR. Serial interval of novel coronavirus (COVID-19) infections. International Journal of Infectious Diseases 93 (2020); 284-286.

19. Pranata R, Huang I, Lim MA, Wahjoepramono EJ, and July Impact of cerebrovascular and cardiovascular diseases on mortality and severity of COVID-19; systematic review, meta analysis, and meta-regression. Journal of Stroke and Cerebrovascular Diseases, Vol. 29, No. 8 (August), 2020: 104949 
20. Huang I, Lim MA, Pranata R. Diabetes mellitus is associated with increased mortality and severity of disease in COVID-19 pneumonia- A systematic review, meta analysis, and meta-regression. Diabetes \& Metabolic Syndrome: Clinical Research \& Reviews; 14 (2020); 395-403.

21. Wu JT, Leung K, Leung GM. Nowcasting and forecasting the potential domestic and international spread of the 2019-nCoV outbreak originating in Wuhan, China: a modelling study. Lancet 2020; 395: 689-97. https://doi.org/10.1016/ S0140-6736(20)30260-9

\section{Tables}

Table 1; Some characteristics of participants in the two groups of COVID 19 patients (primary cases) and their contact cases in the two groups of household and non-household. 


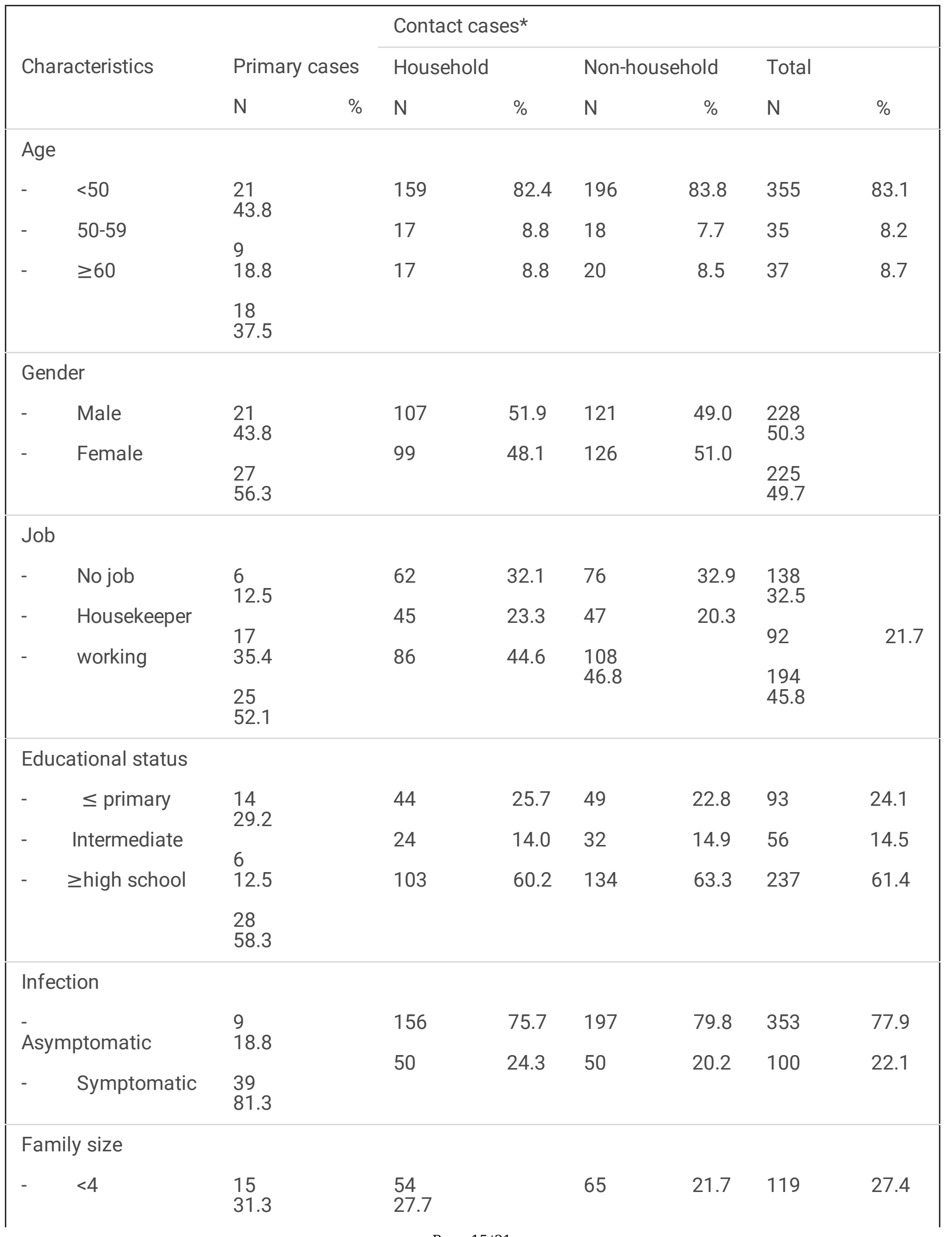


*- In case of some factors, some cases are missing.

Table 2: Frequency distribution of COVID 19 patients (primary cases) based on the type and the mean duration of the first SARS-CoV-2 infection symptom.

\begin{tabular}{|llllll|}
\hline Max & Min & Mean Duration (days) \pm Sd & N & $\%$ & First symptom \\
\hline 12 & 1 & $4.9 \pm 3.3$ & 10 & 20.8 & Fever, chill \\
\hline 28 & 1 & $8.2 \pm 8.6$ & 10 & 20.8 & Cough \\
\hline 12 & 1 & $5.2 \pm 3.9$ & 6 & 12.5 & Body pain \\
\hline 30 & 1 & $8.5 \pm 11.0$ & 6 & 12.5 & Fatigue, weakness, \\
\hline 14 & 2 & $7.0 \pm 6.2$ & 5 & 10.4 & Sore throat \\
\hline 4 & 4 & $4.0 \pm \mathrm{NA}$ & 1 & 2.1 & Headache \\
\hline 1 & 1 & $1.0 \pm \mathrm{NA}$ & 1 & 2.1 & Eye irritation \\
\hline- & - & - & 9 & 18.8 & No symptom \\
\hline 30 & 1 & $6.4 \pm 6.6$ & 48 & 100 & Total \\
\hline
\end{tabular}

Table 3; Secondary attack rates among contact cases in the two groups of household and non-household along with $95 \%$ confidence interval 


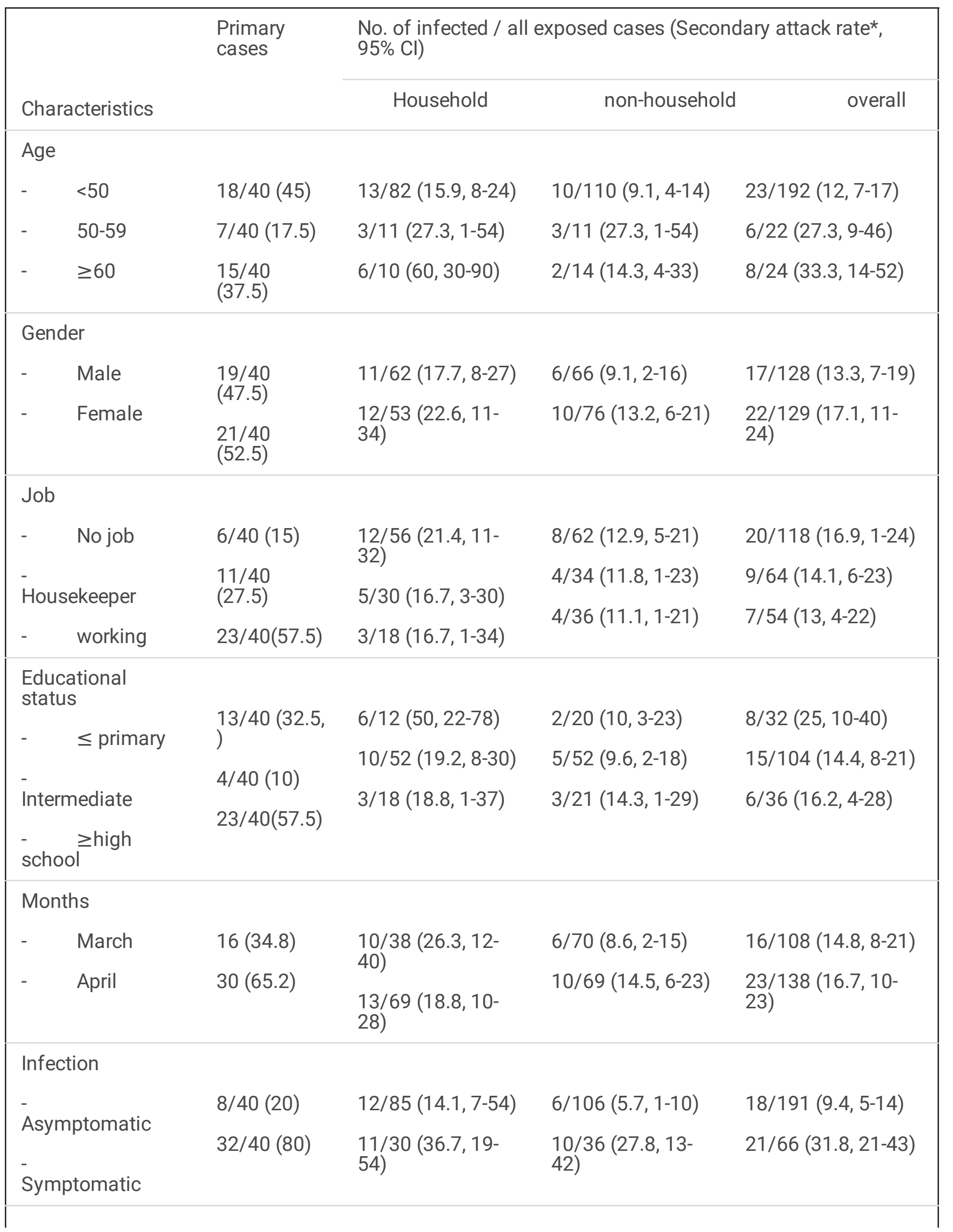


Family size

$\begin{array}{lllll}<4 & 12 / 40(30) & 11 / 28(39.3,21- & 5 / 35(14.3,3-26) & 16 / 63(25.4,15-36) \\ \geq 4 & 28 / 40(70) & 57) & \begin{array}{l}11 / 103(10.7,5- \\ 17)\end{array} & 21 / 179(11.7,7-16) \\ & & 10 / 76(13.2,6-21) & 17)\end{array}$

Data are $\mathrm{n} / \mathrm{N}(\%)$ or secondary attack rate $(95 \% \mathrm{Cl})$. When household was defined on the basis of close relatives (people who are living with the patients in the same house). ${ }^{*}$ Calculated as the number of secondary cases divided by the sum of secondary cases and non-cases.

Table 4; Secondary attack rates among contact cases based on the serological analysis results (IgG titer) and age.

\begin{tabular}{|c|c|c|c|c|c|c|}
\hline \multirow[t]{3}{*}{ Age groups } & \multicolumn{4}{|c|}{ IgG titer } & \multirow{2}{*}{\multicolumn{2}{|c|}{ Total }} \\
\hline & \multicolumn{2}{|c|}{ Negative $(<1)$} & \multicolumn{2}{|c|}{ Positive $(\geq 1)$} & & \\
\hline & $\mathrm{N}$ & $\%$ & $\mathrm{~N}$ & $\%$ & $\mathrm{~N}$ & $\%$ \\
\hline$<10$ & 9 & 100 & 0 & 0.0 & 9 & 100 \\
\hline $10-19$ & 32 & 88.9 & 4 & 11.1 & 36 & 100 \\
\hline $20-39$ & 93 & 86.9 & 14 & 13.1 & 107 & 100 \\
\hline $40-59$ & 51 & 82.3 & 11 & 17.7 & 62 & 100 \\
\hline$\geq 60$ & 16 & 66.7 & 8 & 33.3 & 24 & 100 \\
\hline Total & 201 & 84.5 & 37 & 15.5 & 238 & 100 \\
\hline
\end{tabular}

Chi Square test showed significant difference of IgG positive proportions of different age groups $\left(\chi^{2}=8.2\right.$, $d f=3, p<0.05)$

\section{Figures}




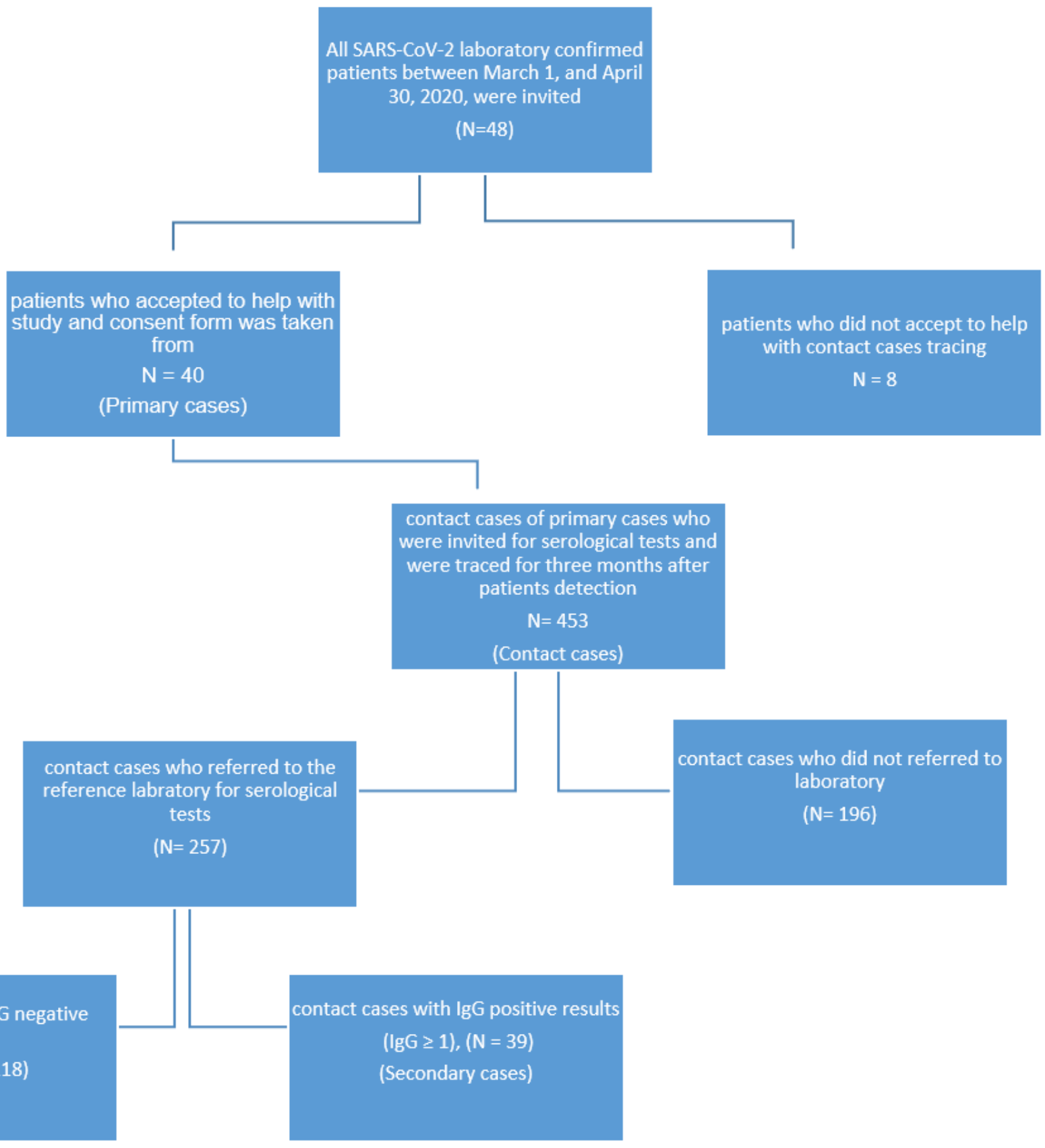

\section{Figure 1}

The flowchart of recruiting SARS-CoV-2 patients and contact cases in the present cohort study. 


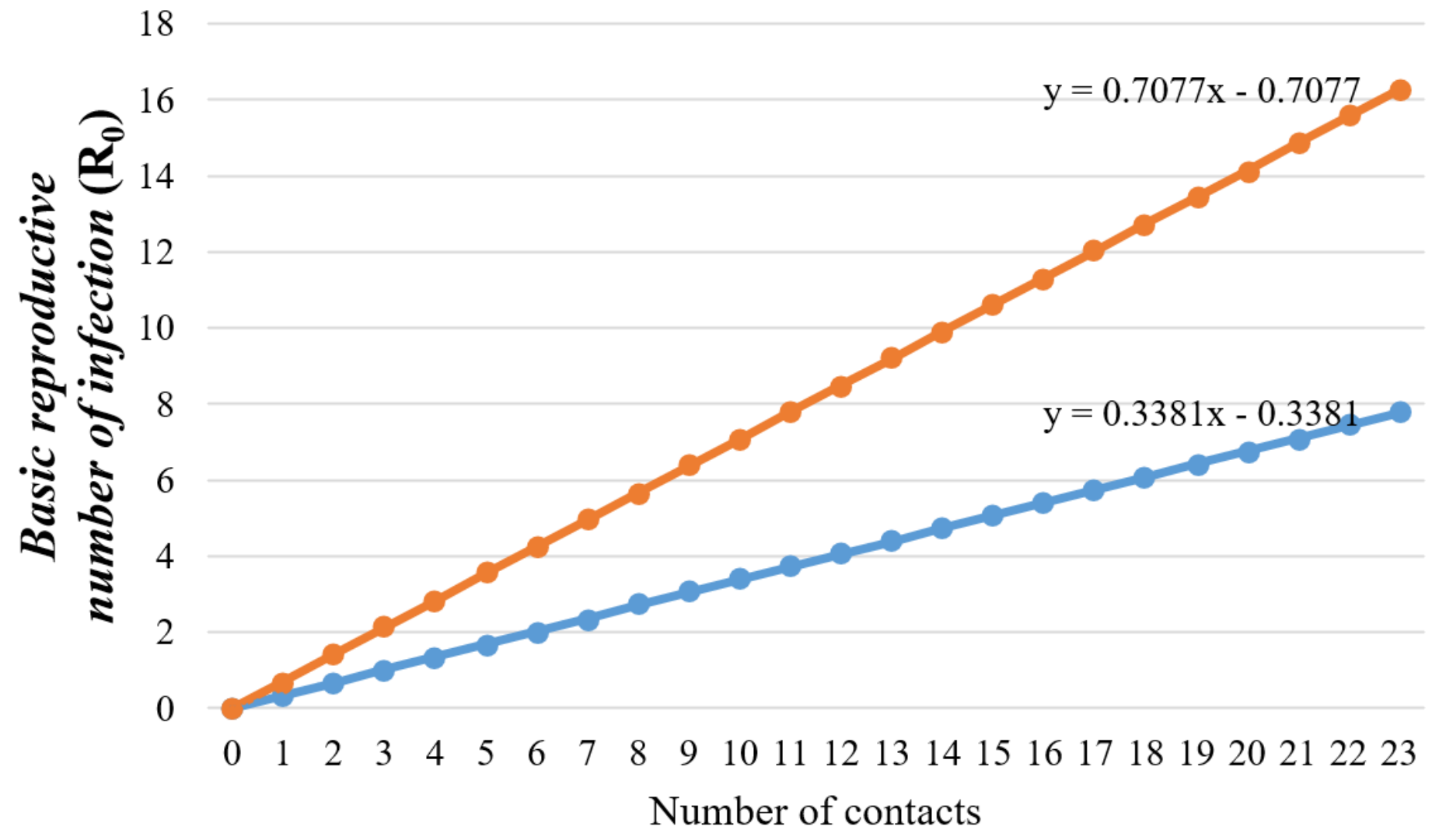

Figure 2

Regression models showing the correlation between R0 values and the number of daily contacts among household (red line) and non-household (blue line) contact cases. 


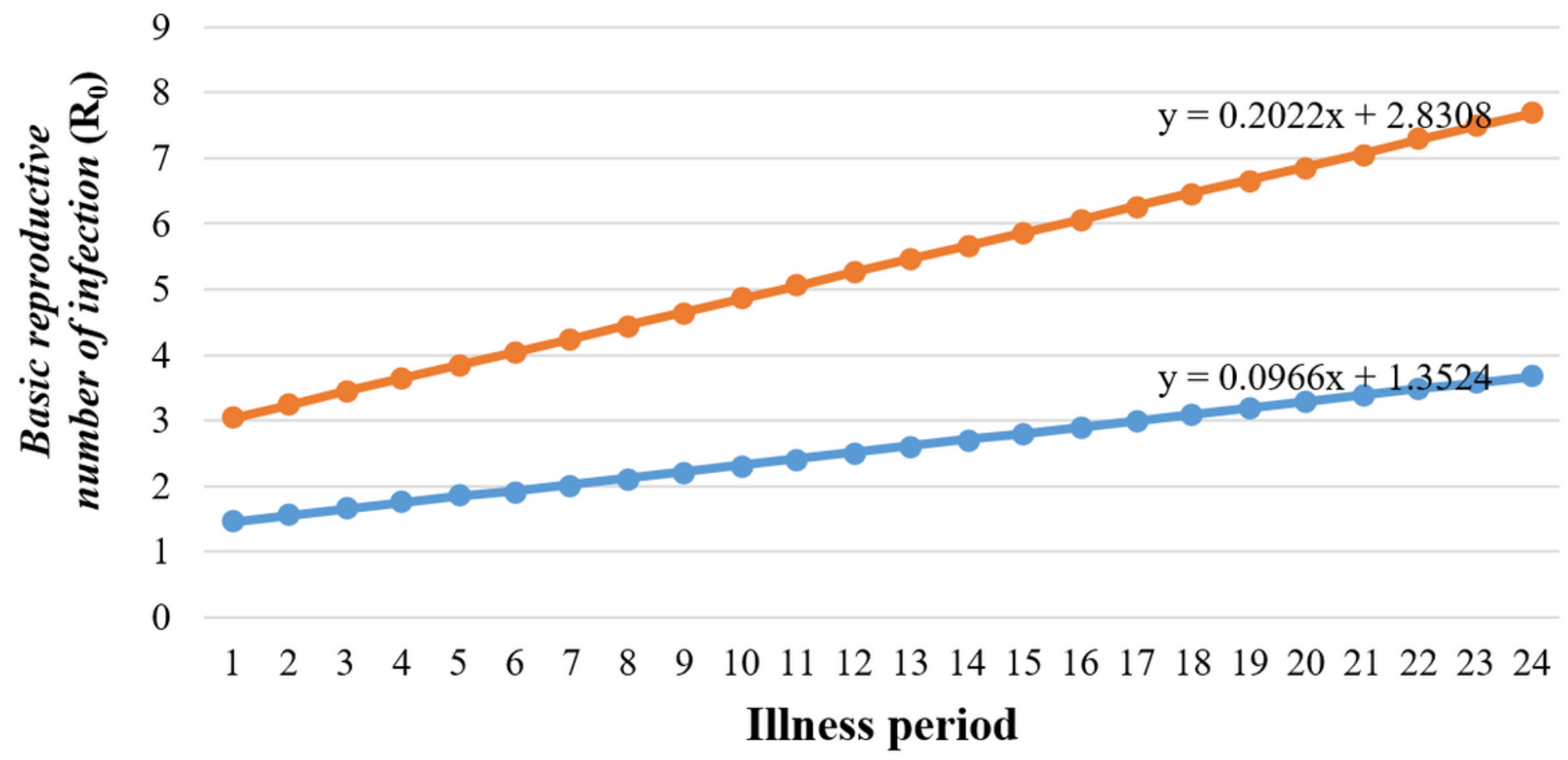

Figure 3

Regression models showing the correlation between R0 values and the duration of symptoms (illness period) among infected cases (days) among household (red line) and non-household (blue line) contact cases. 